\title{
CARTOGRAFIA DE SÍNTESE APLICADA À ANÁLISE DA SEGREGAÇÃO ESPACIAL EM UBERABA, MG
}

\author{
Ricardo Vicente Ferreira \\ Universidade Federal do Triângulo Mineiro, Departamento de Geografia, Uberaba, MG, Brasil \\ ricardo.ferreira@uftm.edu.br \\ Universidade Federal de São Carlos, Curso de Geografia Soberto Martines \\ mmartines@ufscar.br
}

\begin{abstract}
RESUMO
A segregação urbana é um fenômeno social que decorre das desigualdades de acesso à localização e aos bens de consumo coletivo da cidade. Uma maneira de retratar a segregação é através da distribuição espacial dos aspectos econômicos e demográficos da população. O presente estudo constrói um índice de segregação espacial que resume algumas características demográficas urbanas de Uberaba, MG. Faz uso de métodos quantitativos aplicados às cartografia de síntese. Utilizou-se de dados do Censo Demográfico IBGE de 2010, em nível de setores censitários. Ao final, o modelo cartográfico foi validado estatisticamente. Os resultados mostram que, dos 405 setores censitários analisados, $20.83 \%$ apresentam muito alta e alta segregação espacial. Cumulativamente, os índices com média, baixa e muito baixa segregação somam $79,17 \%$, denotando o quantitativo menos crítico. Observa-se que há um gradiente transicional entre os grupos no espaço, indo dos setores censitários mais segregados na periferia aos menos segregados no centro, semelhante ao padrão observado em metrópoles e grandes cidades. O estudo mostra os espaços em condição de segregação, todavia, explicações adequadas destas características requerem a abordagem dos processos históricos políticos que levaram a população que habitam esses espaços.
\end{abstract}

Palavras-chave: Sistemas de Informação Geográfica. Desigualdades. MANOVA.

\section{CARTOGRAPHY OF SYNTESIS APPLIED TO THE ANALYSIS OF SPATIAL SEGREGATION IN UBERABA, MG}

\begin{abstract}
Urban segregation is a social phenomenon resulting from inequalities in relation to the location and the goods in the city. A manner to portray segregation is consider the spatial distribution of the economic and demographic aspects of the population. The present study builds an index of spatial segregation that summarizes some urban demographic characteristics of Uberaba, MG. Quantitative methods are used and applied to cartography of synthesis. Were used data from the 2010 IBGE Demographic Census Tracts. At the end, the cartographic model was statistically validated. The results show that, from the 405 sectors analyzed, $20.83 \%$ have high and very high spatial segregation. Cumulatively, the indexes with medium, low and very low segregation add up to $79.17 \%$, denoting a less critical quantitative. There is a transactional gradient between groups in space, going from more segregated census tracts in the periphery to less segregated in the center, similar to the pattern observed in metropolitans and big cities. The study shows the spaces in a condition of segregation, however, adequate explanations of these characteristics require the approach of the historical and political processes that led these populations to inhabit these spaces.
\end{abstract}

Keywords: Geographic Information System. Inequalities. MANOVA.

\section{INTRODUÇÃO}

O conceito de segregação socioespacial denota a ideia de separação espacial de populações no espaço urbano. Tal diferenciação coloca as populações em condições diferenciadas em relação ao acesso a bens de consumo e serviços disponíveis na cidade. Segundo Vieira e Melazzo (2003) 
existem diferentes visões acerca dos fatores explicativos da segregação urbana, contudo, os pensamentos convergem para a ideia de que a segregação urbana é um fenômeno social e decorre das desigualdades de acesso à localização e aos bens de consumo coletivo da cidade.

A forma como a segregação socioespacial reflete as desigualdades na cidade implica em conhecimento de como as relações sociais foram sendo construídas ao longo do tempo, e as implicações sobre o acesso ao uso da terra, à formulação e promoção de políticas públicas e as relações de sociabilidade e identidade que colocam cada grupo social em determinadas regiões do espaço urbano (VIEIRA; MELAZZO, 2003; YAO et al., 2018). Tratam-se que questões que envolvem processos subjetivos, portanto, as explicações se fazer com base na interpretação da dinâmica das políticas públicas, da organização das classes sociais e do acesso aos espaços da cidade no tempo. Segundo Lefebvre (2001), a segregação espacial ocorre de três formas simultâneas e sucessivas: espontânea, voluntária e programada, com segregação espontânea resultante do preço da terra e, consequentemente, da capacidade das pessoas de arcar com os custos de moradia em determinadas localidades da cidade; segregação voluntária refere-se à autossegregação espontânea, como em condomínios residenciais e a segregação programada, relacionada à ação estatal no planejamento habitacional.

Uma maneira alternativa de retratar a cidade no âmbito da segregação sociespacial é entender o grau de segregação em um determinado momento. Esta análise pode ser elaborada com base na avaliação das características da sociedade, expressas pelo perfil demográfico. Assim, a abordagem se faz com base em medidas que resumem aspectos da sociedade que tem relação com a segregação e que, ao mesmo tempo, podem ser espessos espacialmente.

No contexto da análise da segregação espacial, as características de uma população podem ser resumidas em índices, desde que os aspectos econômicos e demográficos sejam expressos por variáveis organizadas e níveis de medidas, tais como, idade, renda, gênero, escolaridade, etc. (GOERL et al., 2012) e resultem em um modelo de entendimento da dinâmica espacial dos grupos de acordo com características socioeconômicas e demográficas, possibilitando a análise da conjuntura que tal população está submetida (CUNICO; OKA-FIORI, 2014).

Yao et al. (2018) coloca que a análise espacial de segregação feita por meio de medidas, geralmente resulta em formação de agrupamentos em determinadas regiões da cidade, com verificação de diferentes níveis de valores que podem ser relacionados a intensidade da separação espacial que definem os grupos sociais. Estes métodos são construídos com base em dados agrupados por unidades administrativas dos censos demográficos.

Nos debates atuais sobre o desenvolvimento de índices aplicados à segregação espacial, Wong (2016) descreve que o espaço, embora não seja a única dimensão da segregação, é um aspecto importante de análise, contudo, para a melhor aproximação da realidade, algumas questões dever ser consideradas, como à incorporação de estatísticas espaciais e o nível da escala de agrupamento dos dados mapeados. O problema relacionado a esta segunda questão reside na mistura de diferentes grupos populacionais em uma mesma unidade territorial recenseada. Disso decorreram indagações sobre a validade das explicações dos agrupamentos observados nos mapeamentos urbanos feitos com base em setores censitários. Em linhas gerais, chamam a atenção para a interpretação dos agrupamentos, centralidades e uniformidades observadas nos mapeamentos de índices de segregação espacial, de modo a não incorrer em erros de interpretação. Todavia o autor deixa claro que para a que uma medida seja eficaz, ela precisa ser sensível ao arranjo espacial das distribuições populacionais. Ou seja, quando as características que resumem o perfil de uma população mudam, o índice consegue capturar, no espaço, os diferentes grupos populacionais relacionados a essas mudanças, reforçando o princípio da dependência espacial do fenômeno.

O padrão de segregação social observado nas cidades brasileiras está vinculado aos processos históricos de urbanização de grande e médias cidades no país (SANTOS, 2005), e se materializa em diferentes padrões no espaço das cidades. O padrão característico nas grandes cidades brasileiras é a divisão centro versus periferia, onde o centro dispõe de mais serviços urbanos, públicos e privados e é ocupado pelas classes de mais alta renda, e a periferia que é subequipada e distante do centro, sendo ocupada pelas classes de mais baixa renda (VILLAÇA, 1998).

O presente estudo faz uma análise da segregação espacial da cidade, com base na construção de um índice que resume sete características demográficas e ambientais do espaço urbano de Uberaba, MG. Adota como referencial metodológico a Cartografia de Síntese, construída por técnicas de análise espacial em Sistemas de Informação Geográfica. Ao final, propõe um índice de segregação espacial e valida estatisticamente os resultados do modelo. 


\section{Área estudada}

O município de Uberaba está localizado na região do Triângulo Mineiro e Alto Paranaíba, no Estado de Minas Gerais. Seu perímetro urbano tem cerca de $309 \mathrm{~km}^{2}$, segundo o plano diretor da Prefeitura Municipal de Uberaba de 2018, porém a superfície da macha urbana perfaz cerca de $64 \mathrm{~km}^{2}$, estimada por sensoriamento remoto. Em 2010 a divisão territorial do IBGE definia 36 subdistritos do tipo urbano (Figura 1)Caracteriza-se como uma cidade média, que desde a década de 1970 tem ampliado seu espaço urbano devido às mudanças produtivas, sobretudo, decorrentes da modernização do campo, tendo com aporte importante da presença da agroindústria da cana de açúcar e atividades industriais neste processo. Uma das consequência desta modernização para a cidade foi o surgimento de novos serviços, que passaram a reorganizar o espaço urbano através do estabelecimento de shoppings centers, hipermercados, lojas de departamentos e centros comerciais, resultando em uma reestruturação dos negócios imobiliários. Essa nova configuração foi acelerada a partir da década de 2000 (GOMES, 2015). Em decorrência destas mudanças, a população urbana aumentou de 108.259, em 1970 para 289.376 habitantes em 2010, representando 97,7\% da população total do munícipio, segundo dados do IBGE em 2010.

Figura 1 - Município de Uberaba. (a) posição no território brasileiro com destaque para o estado de Minas Gerais e Região do Triângulo Mineiro e Alto Paranaíba; (b) Território municipal com destaque para o perímetro urbano e subdistritos urbanos (IBGE, 2010); (c) contorno dos subdistritos urbanos e imagem do sensor RapidEye (composição: b1 azul; b2 verde, b3 vermelho) datada de 25/05/2014 com destaque para a área urbana ocupada.

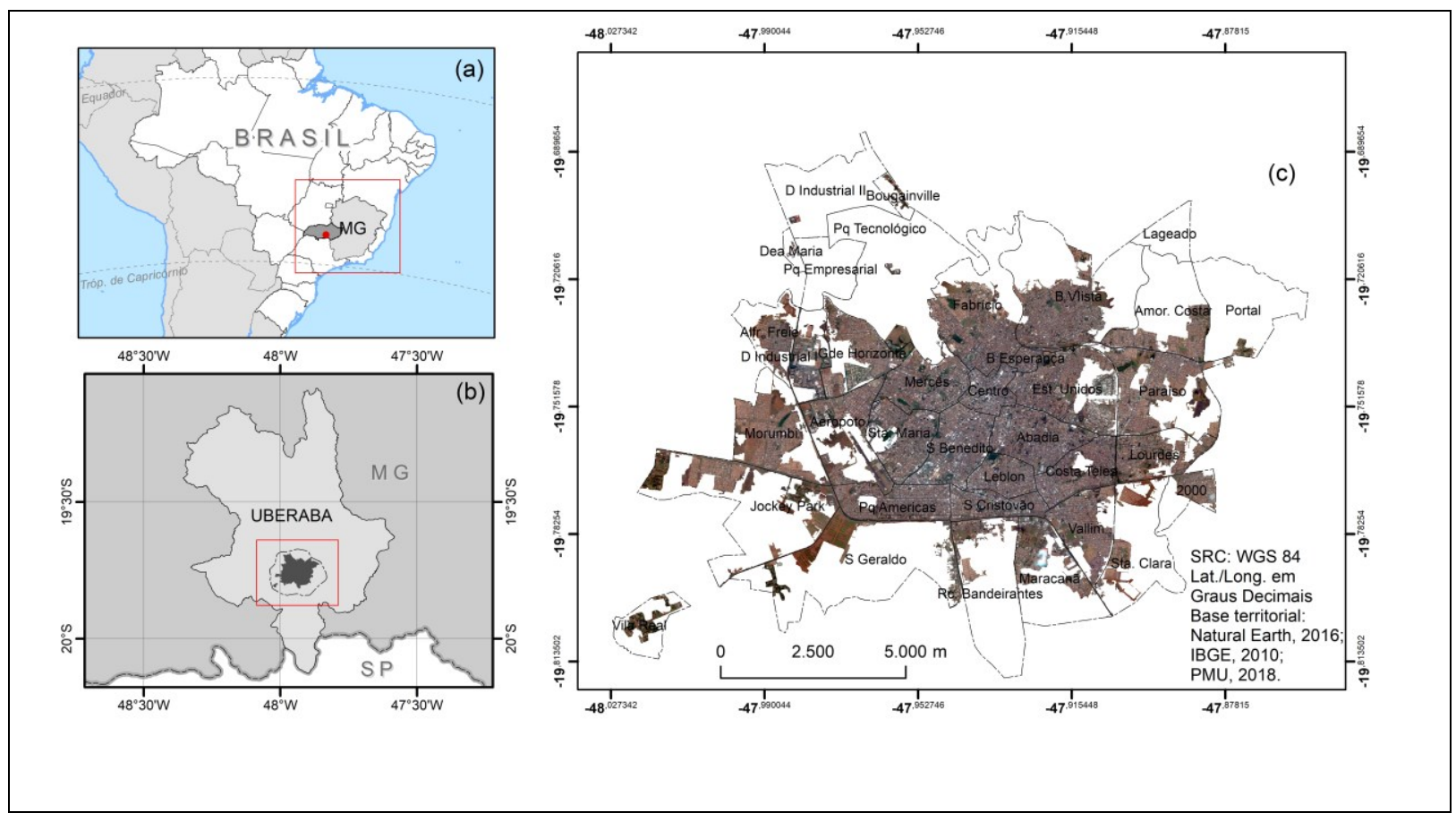

\section{MATERIAIS E MÉTODOS}

Os recursos utilizados para a execução deste estudo foram: dados do Censo Demográfico IBGE (2010 disponibilizados em planilhas eletrônicas, polígonos vetoriais dos setores censitários e imagem multiespectral do Sensor Rapideye com passagem em 25/02/2014. Os softwares utilizados para mapeamento foram: ArcGis, versão 10.6 e QGIS, versão 3.8. Para o tratamento e análises estatísticas foram utilizados o softwares Excel 2013 e Past, versão 4.0.

O estudo se baliza em metodologia da cartografia de análise de síntese, onde vários atributos que tem relação com um determinado tema são reunidos e analisados em conjunto (MARTINELLI, 2018). Esse método permite salientar as relações espaciais entre as diferentes variáveis postas em análise. Na síntese não se tem mapas em sobreposição, mas sim, a identificação de agrupamentos que resumem os valores ou níveis das variáveis postas em observação e que podem ser analisadas por meio de procedimentos estatístico-matemáticos, pois estes permitem resultados mais objetivos, em contraposição aos métodos que fazem uso da interpretação visual. Assim, adota-se a análise denominada multivariada, tratando quantitativamente os diversos atributos contidos nas distintas variáveis geográficas (QUEIROZ FILHO; MARTINELLI, 2007). 
Ao longo do desenvolvimento da pesquisa foram seguidas as seguintes etapas: 1) definição das variáveis para a composição do índice de segregação espacial agrupadas por setores censitários; 2) elaboração do modelo dasimétrico para a área urbana de Uberaba; 3) normalização das variáveis e elaboração de um índice sintético de avaliação da segregação espacial; 4) validação estatística do índice.

\section{Variáveis que compõe o índice de segregação espacial}

Para esse estudo foi assumida como unidade espacial de análise os setores censitários definidos no censo IBGE de 2010. O município de Uberaba possui 409 setores censitários do tipo urbano, 4 (quatro) setores não foram considerados por não possuírem dados disponíveis, assim, o universo de análise da pesquisa se resumiu a 405 setores censitários.

O critério da seleção das variáveis para compor o índice de segregação espacial se baseou na metodologia adotada pela Fundação Seade na elaboração do Índice Paulista de Vulnerabilidade Social (FUNDAÇÃO SADE, 2010) e pelo Atlas da vulnerabilidade social nos municípios brasileiros (COSTA; MARGUTI, 2015). Dentre os critérios que nortearam estes dois estudos, destaca-se a segregação espacial como um fenômeno presente nos centros urbanos e que podem ser estudados com base em três categorias de dados levantados pelo censo demográfico do IBGE: (1) variáveis socioeconômicas, como renda e escolaridade; (2) variáveis relacionadas ao ciclo de vida familiar, como a presença de crianças, chefes de família muito jovens ou muito idosos; (3) e as variáveis que retratam as condições habitacionais, expresso nas condições de saneamento, coleta de lixo e abastecimento de água. Assim, entendeu-se que estas variáveis são também representativas para a construção do índice municipal para a cidade de Uberaba, tendo como referência resultado da amostra por setores censitários do Censo IBGE de 2010. Foram selecionadas as seguintes variáveis para compor o índice:

v1. Rendimento nominal do responsável pelo domicílio: Razão entre o valor do rendimento nominal médio mensal de responsáveis por domicílios particulares permanentes pelo número total de responsáveis por domicílio;

v2. Responsável por domicílio entre 10 e 29 anos por setor: Razão obtida através do somatório das pessoas de 10 a 29 anos responsáveis pelos domicílios e o total de pessoas do setor censitário. Esses dados expressam a proporção dos jovens que ocupam a posição de chefes de família;

v3. Responsável por domicílio com idade igual ou maior que 60 anos: Razão entre o número total de pessoas responsáveis por setor com mais de 60 anos pelo número total de pessoas responsáveis pelo domicílio;

v4v. Responsável alfabetizado por domicílio por setor: Razão entre o número total de pessoas responsáveis alfabetizadas por setor, pelo número total de pessoas responsáveis pelo domicílio;

v5. Crianças entre 0 e 5 anos por setor censitário: Razão obtida pelo somatório das pessoas entre 0 e 5 anos e o número total de pessoas. Esses dados capturam a proporção de crianças dentro dos setores censitários:

v6. Domicílios quitados: Razão do número de domicílio integralmente pago pelo número total de domicílios no mesmo setor censitário;

Os dados referentes ao saneamento básico não foram considerados pois, para a área urbana não foi verificado uma variabilidade significativa entre os dados que expressasse uma diferenciação espacial entre os setores censitários. A média para o esgotamento sanitário foi de $92,7 \%$ e coeficiente de variação (C.V.) de 0.26, o abastecimento de água $92.3 \%$ e C.V. 0,27 e a coleta de lixo 95,3\% e C.V. 0,21. Contudo, foi adicionada uma variável ambiental auxiliar relacionada a estimativa da arborização urbana. A adoção de tal critério se alinha ao princípio de que a baixa qualidade ambiental nas cidades resulta de problemas socioambientais, sendo um dos indicadores a escassez de áreas verdes (CUNICO; OKA-FIORI, 2014). Assim, essa variável compôs o sétimo critério adotado neste estudo, a saber:

v7. Área arborizada estimada; razão da área com cobertura de vegetação arbórea estimada com a área dasimétrica do setor censitário.

\section{Elaboração do modelo dasimétrico para a área urbana de Uberaba}

O levantamento dos dados pelo IBGE (2010) adota como a menor unidade territorial municipal os setores censitários e são classificados em função de seus usos como: urbano, mistura de usos urbano e/ou rural. Neste estudo foi considerado apenas o espaço urbano ocupado. Assim, as feições territoriais dos setores

$\begin{array}{lllll}\text { Caminhos de Geografia } \quad \text { Uberlândia-MG } & \text { v. 22, n. } 80 \quad \text { abr./2021 } & \text { p. 297-308 } & \text { Página } 300\end{array}$


censitários foram recortadas com base na identificação da mancha urbana observada em imagem de satélite. Esse procedimento é denominado método dasimétrico (WRIGHT, 1936). Trata-se de um tipo especifico de interpolação zonal, baseada em técnica cartográfica que utiliza informações auxiliares para desagregar variáveis demográficas, por exemplo, população total e densidade populacional (FRANÇA et al., 2014).

Para a obtenção da dasimetria foi estimada a mancha urbana a partir de técnicas de sensoriamento remoto, com uso de imagem do Sensor Rapideye, com passagem de 25/05/2014. A aplicou-se sobre as bandas banda 2 (verde): 520 - $590 \mathrm{~nm}$, banda 3 (vermelho): 630 - $685 \mathrm{~nm}$ e banda 5 (infravermelho próximo): 760 - $850 \mathrm{~nm}$ o classificador de máxima verossimilhança (MaxVer, na imagem (Dados da imagem). O Maxver é um algoritmo de classificação da máxima verossimilhança que é um classificador paramétricos de método supervisionado (INPE, 2008). Foram definidas as classes de separação de usos e coberturas de modo a isolar a mancha urbana dos demais tipos de usos. O resultado da classificação foi submetido ao filtro Majority Filter de modo a eliminar pixel isolados. Em seguida o arquivo raster classificado foi convertido para o modelo vetorial, e selecionados apenas os polígono classificados como urbano. Os polígonos dos setores censitários foram, então, recortados com base nas feições urbanas dasimétricas, reduzindo-se assim as dimensões dos polígonos censitários contendo baixa ocupação. Por meio deste procedimento obteve-se polígono censitários efetivamente ocupados na mancha urbana.

\section{Estimativa da arborização urbana}

A imagem do sensor Rapideye foi também utilizada para a estimativa da arborização urbana. O procedimento foi realizado pelo Índice de Vegetação pela Diferença Normalizada (NDVI). O índice é calculado com base na refletância no espectro eletromagnético vermelho $(630-685 \mathrm{~nm})$ e infravermelho próximo (760-850nm) (ROUSE et al., 1974). A partir do índice foi identificado, por interpretação visual, o limiar que melhor isolou a cobertura arbórea urbana, no caso os valores entre 0,350 e 0,677 (Figura 2 . Essa faixa de valores de pixels do NDVI foi reclassificada e transformada para o modelo vetorial. Os polígonos representativos da superfície arbórea estimada foram interseccionados aos polígonos dasimétricos dos setores censitários. Por fim, calculou-se a razão de área arbórea estimada para a superfície de cada setor censitário.

\section{Normalização das variáveis e elaboração do índice de segregação espacial}

As variáveis que foram consideradas para a elaboração índice de segregação espacial são documentadas em diferentes grandezas numéricas. Assim, os esses atributos foram padronizados para uma escala numérica de 0 a 1 (Equação 1) (MARTINES et al., 2017). Os setores censitários com valores próximos a 1 revelaram as melhores condições de cada indicador, enquanto que os valores próximos a 0 indicam as piores situações. A normalização das variáveis: rendimento nominal do responsável pelo domicílio;

domicílios quitados; responsável alfabetizado por domicílio; área arborizada estimada considerou que quanto menor o valor, maior é o indicativo de segregação, vice versa. Portanto, a seguinte equação foi aplicada.

$$
\operatorname{Var}=\left(\left(x_{i}-\min \right) /(\max -\min )\right)
$$

Onde:

Var = valor resultado da normalização por setor censitário;

$\mathrm{x}_{\mathrm{i}}=$ valor na posição $\mathrm{i}$ no conjunto de dados da variável a ser normalizada;

$\min =$ menor valor no conjunto de dados da variável considerada;

max = maior valor no conjunto de dados da variável considerada;

Com relação às variáveis: responsável por domicílio entre 10 e 29 anos; responsável por domicílio com idade igual ou maior que 60 anos; crianças entre 0 e 5 anos, o critério é oposto, quanto menor o valor, menor o indicativo de segregação, vice versa. Assim, a normalização foi feita pela aplicação da seguinte equação:

(Eq.2)

$$
\operatorname{Var}=\left(\left(\max -x_{i}\right) /(\max -\min )\right)
$$


Para a obtenção do índice de segregação espacial foi aplicada a seguinte equação. (Eq.3)

Eq.3.

$$
\mathrm{SE}=\sum(\operatorname{Var})
$$

Onde:

SE = valor do índice de segregação espacial por setor censitário;

Var = variável selecionada normalizada .

Como a obtenção do índice de segregação espacial aplicou-se a regra de stuges (Eq.4) (SCOTT, 2009) que permite obter o número de classes que são representativas de uma distribuição. Esse procedimento gerou a indicação de 5 classes para representar a distribuição dos dados do índice.

$$
K=1+3,3 \cdot \log n
$$

Onde:

$\mathrm{K}=$ número de classes;

$\mathrm{n}=$ número total de informações

A partir da definição das 5 classes foi adotado o método de quebras naturais (JENKS, 1967), que identifica as grandes lacunas no conjunto de valores, ou as pausas naturais, maximiza a diferença entre as classes e minimiza as diferenças dentro das classes. Para essas classes foi atribuída a nomenclatura qualitativa: Muito alta, Alta, Média, Baixa, Muito baixa.

\section{Validação estatística do índice}

No processo de elaboração do índice de segregação apresentado foi elaborado a partir de diversas características econômicas, sociais e demográficas da população urbana, entretanto se faz necessário estabelecer um processo de verificação e validação, de modo a identificar possíveis inconsistências entre os agrupamentos de foram definidos para tipificar os setores censitários, assim foram aplicados os seguintes procedimentos estatísticos:

Inicialmente aplicou-se uma análise descritiva por boxplot e jitterplot, para avaliar a distribuição empírica dos dados e a distribuição dos valores de cada parâmetro em relação aos grupos de variáveis estabelecidos. Em seguida, realizou-se o teste hipótese pela Multiple Analysis Of Variance (MANOVA), para revelar possíveis diferenças entre o conjunto de variáveis selecionados dentro de cada grupo e entre os grupos. Esse teste é realizados a partir do calcula o lamdba $(\Lambda)$ de Wilks, que é uma estatística de teste da MANOVA. O teste define se existem diferenças entre médias de grupo para uma combinação particular de variáveis dependentes e se materializa no teste $F$, que deve ser maior que 1, e o valor-p (same) deve ser menor que 0,05 (TODOROV; FILZMOSER, 2010).

O ultimo procedimento estatístico foi a aplicação da análise multivariada de componentes principais (PCA), o que propicia avaliar a importância de cada parâmetro em relação aos grupos definidos a partir do índice de segregação espacial urbana. A PCA permitiu analisar o conjunto de variáveis utilizados no índice e reduzir as sobreposições dos parâmetros adotados na composição do índice.

\section{RESULTADOS}

Cada tema utilizado na construção do índice normalizado foi mapeado individualmente, onde foi possível uma interpretação visual dos resultados tendo como referência o recorte dasimétrico e a sobreposição da divisão territorial dos 36 subdistritos definidos pelo IBGE em 2010 (Figura 2). A distribuição espacial dos índices correspondentes ao Rendimento nominal do responsável pelo domicílio, Responsável por domicílio entre 10 e 29 anos; Responsável por domicílio alfabetizados, Crianças entre 0 e 5 anos e Domicílios quitados por setor censitário denotam um padrão centro-periferia, contudo, cada qual descrevendo uma variação específica de suas respectivas intensidades e faixas de agrupamento. Os subdistritos das bordas leste, sudeste e oeste são os que apresentam tendência aos baixos índices destes temas, ou seja, indicam maior segregação espacial. Com relação ao índice Responsável por domicílio acima de 60 anos verifica-se a tendência ao padrão inverso, no centro e subdistritos adjacentes os valores mais baixos do índice indicam haver maior proporção de idosos como responsáveis pelo domicílio. A distribuição espacial da taxa de arborização indica ausência de agrupamento ou padrão de distribuição. 
Figura 2 - Distribuição espacial do índice de cada variável temática adotada na construção do modelo. Valores baixos indicam pior condição, valores altos melhor condição. (v1) Rendimento nominal do responsável pelo domicílio; (v2)

Responsável por domicílio entre 10 e 29 anos por setor; (v3) Responsável por domicílio com idade igual ou acima de 60; (v4) Responsável por domicílio alfabetizados por setor; (v5) Crianças entre 0 e 5 anos por setor censitário; (v6)

Domicílios quitados; (v7) Taxa de arborização estimada.
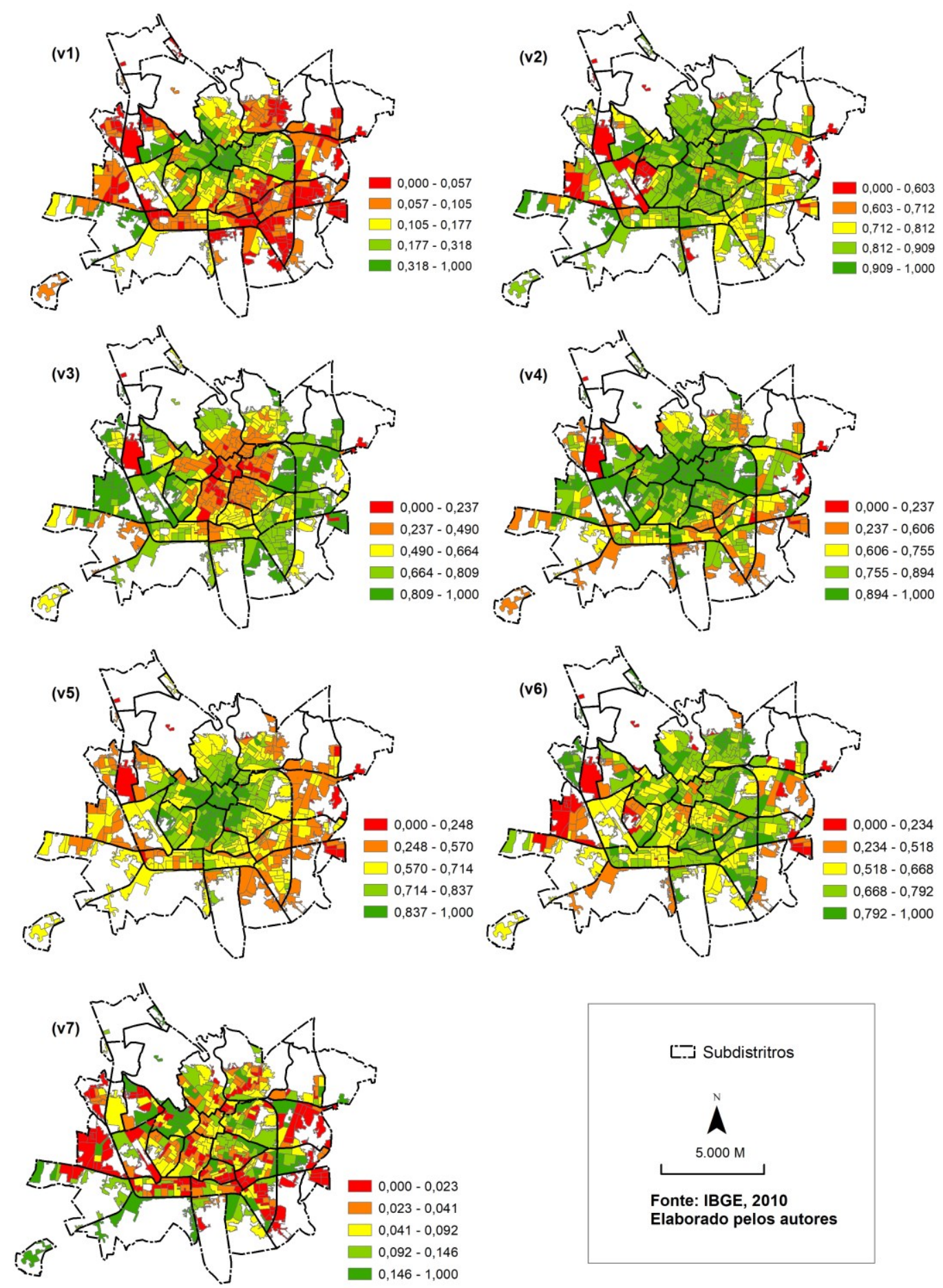

A Tabela 1 apresenta a distribuição de frequência dos índice de segregação espacial por classificação temática em função dos setores censitários, os dados demonstram que $20.83 \%$ dos setores considerados formam classificados com muito alta e alta segregação e 32.60 em estágio de média. Apenas 11.52 dos setores apresentam muito baixo índice de segregação. Cumulativamente, os índices com média, baixa e muito baixa segregação somam $79,17 \%$, denotando o quantitativo menos crítico no território urbano.

$\begin{array}{lllll}\text { Caminhos de Geografia } \quad \text { Uberlândia-MG } & \text { v. 22, n. } 80 & \text { abr./2021 } & \text { p. 297-308 Página } 303\end{array}$


Tabela 1 - Distribuição de Frequência dos setores censitários por classificação da segregação espacial.

$\begin{array}{lcc}\text { Classe } & \text { Frequência } & \% \\ \text { Muito alto } & 25 & 6.13 \\ \text { alto } & 60 & 14.71 \\ \text { médio } & 133 & 32.60 \\ \text { baixo } & 143 & 35.05 \\ \text { muito baixo } & 47 & 11.52 \\ \text { Total } & \mathbf{4 0 8} & \mathbf{1 0 0 . 0 0}\end{array}$

A combinação dos temas indicados na Figura 2 resultou em um mapa com a síntese dos índices (Figura 3). O agrupamento do índice em 5 classes temáticas pelo método das quebras naturais (Jenks) monstra que predomina o modelo centro-periferia, sendo os subdistritos das extremidades leste e oeste da cidade os que indicam maior segregação espacial. Isso pode ser explicado pelo fato de estes serem espaços de ocupação mais recente, e ter uma relação com o preço da terra ser mais baixo capacitando estas populações a arcarem com os custos de moradia nestas localidades menos centrais. Alguns enclaves com maior segregação aparecem mais ao centro, a exemplo dos bairros Abadia, Santa Maria e Boa Vista. Tal fato denota indicativos de desigualdade socioeconômica nestes setores, contudo, tal segregação é resultante do processo de formação destes espaços ao longo do tempo que, de alguma forma, conduziu uma parcela da população a ocuparem estes locais.

Figura 3 - Mapa síntese do índice de segregação espacial da cidade de Uberaba, MG.

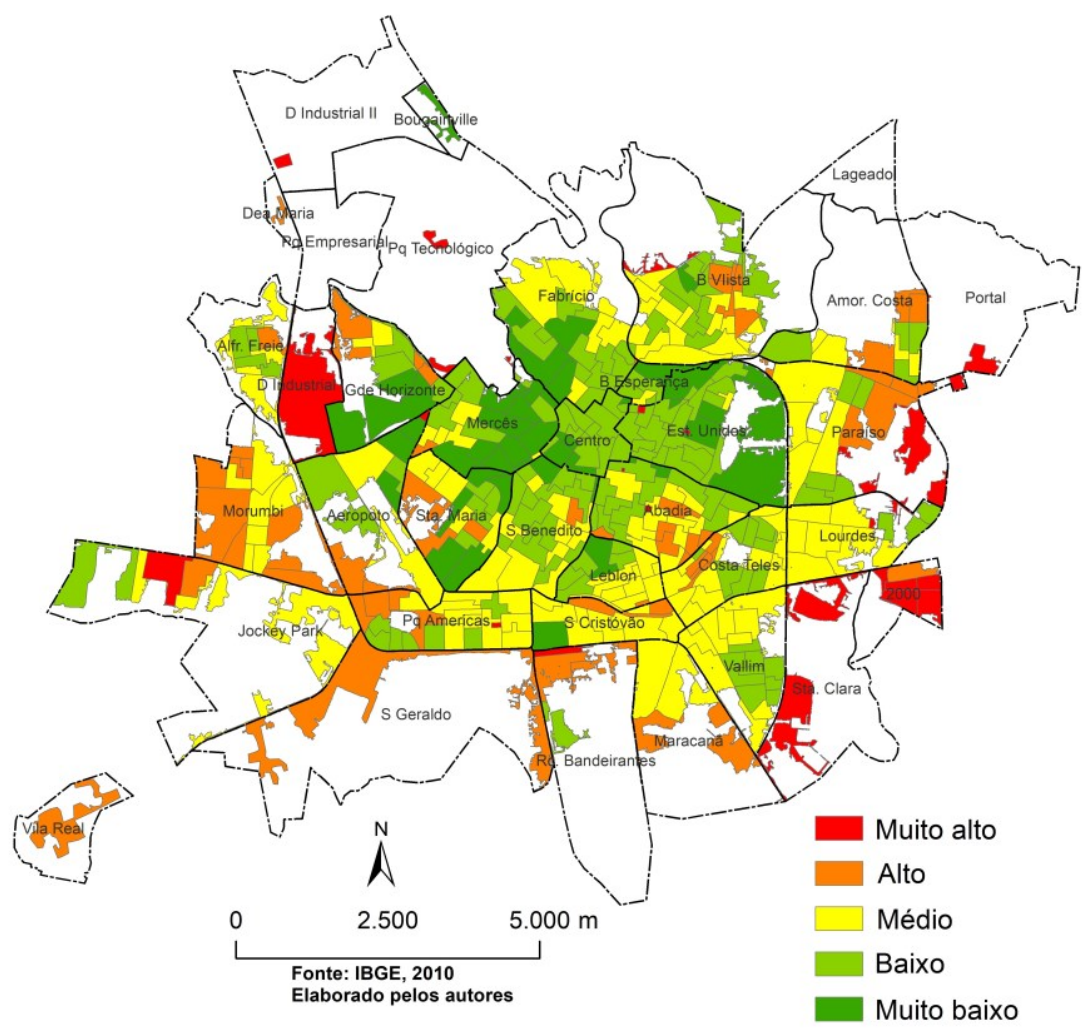

A distribuição dos dados no histograma tem um padrão assimétrico negativo, com maior frequência e dos entre a média $(\mu)$ e o primeiro desvio padrão $(\sigma)$ acima da média (Figura 4). Assim, há predominância de setores censitários com nível de segregação espacial média e baixo. A média $(\mu)$ está na segregação espacial de nível médio e, a partir desta, até o primeiro desvio padrão abaixo concentram-se a maior frequência de setores censitários como alta segregação espacial. Uma atenção deve ser dada aos setores de alta segregação espacial, que se concentram em uma posição muito a abaixo das faixas predominantes. 
Figura 4 - Histograma de frequência do índice por setor censitário dasimétrico na cidade de Uberaba, MG.

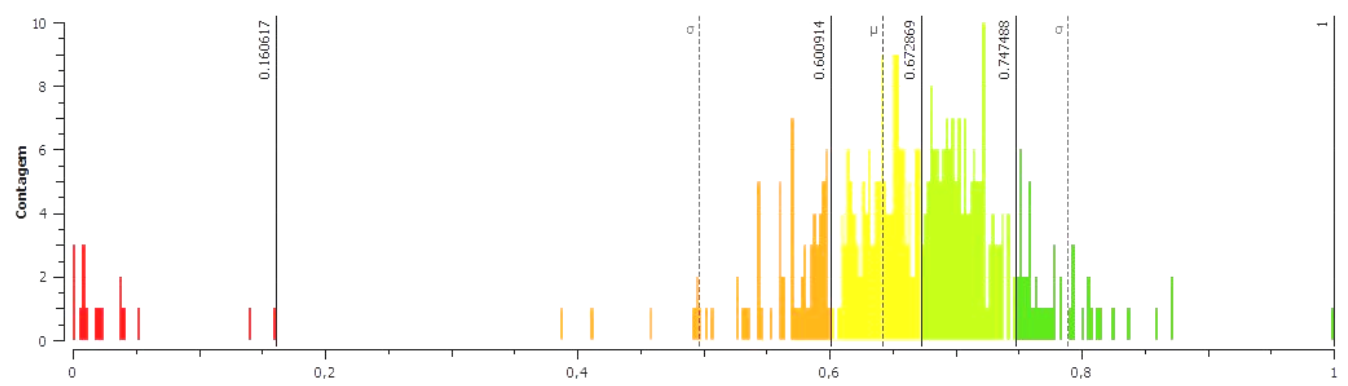

A Figura $5 a$ apresenta a distribuição dos valores do índice para cada variável na forma de gráfico, um destaque particular é dado à variável renda (v1), com grande dissimilaridade nos dados e apresentando valores discrepantes a partir de $50 \%$ quando observada a distribuição do índice por setores censitários, isso mostra que poucos setores tem alta média de renda (Figura 5a). Em contrapartida, a maioria dos setores está concentrada nos níveis a partir de $20 \%$, essa constatação também pode ser confirmada no Gráfico Boxplot (Figura $5 \mathrm{~b}$ ), onde observa-se um grande alongamento do gráfico no $4^{\circ}$.quartil, confirmando a dissimilaridade nessa variável (v1).

As distribuição do índice por setores censitários é diferente para cada tema, no entanto, as variáveis v1 e v7 tem um padrão semelhante, com algumas variáveis tem um padrão de distribuição semelhantes. A influência de responsáveis jovens (v2) e de idosos (v3) na responsabilidade da chefia dos domicílios, indicam que os setores com a maior segregação espacial, são os que tem os índices concentrados a partir da mediana, esse comportamento é confirmado na dispersão do gráfico Boxplot (Figura 5b) que apresenta dissimilaridade nas variáveis v2 e v3 no $1^{\circ}$ quartil. A maior concentração de crianças entre 0 e 5 anos (v5), pressupõe maior demanda sobre o setor público no provimento de educação e saúde, apresenta os setores censitários mais segregados com os menores índices (Figura 5a,b), que apresenta o grau de dissimilaridade no $1^{\circ}$ quartil. Quanto ao índice de arborização (v7), nota-se a concentração na base do gráfico (Figura 5a), isso está relacionado com o forte adensamento e impermeabilização do solo nas áreas urbanas centrais, o que tem como consequência a subpressão da vegetação arbórea.

Figura 5 - Distribuição gráfica do comportamento das variáveis que compõem o índice de segregação espacial. (a) Jitterplot do índice por variável (b) Boxplot da distribuição do índice por variável; sendo: (v1) Rendimento nominal do responsável pelo domicílio; (v2) Responsável por domicílio entre 10 e 29 anos por setor; (v3) Responsável por domicílio acima de 60 anos e mais; (v4) Responsável por domicílio alfabetizados por setor; (v5) Crianças entre 0 e 5 anos por setor censitário; (v6) Domicílios quitados; (v7) Taxa de arborização.

(a)

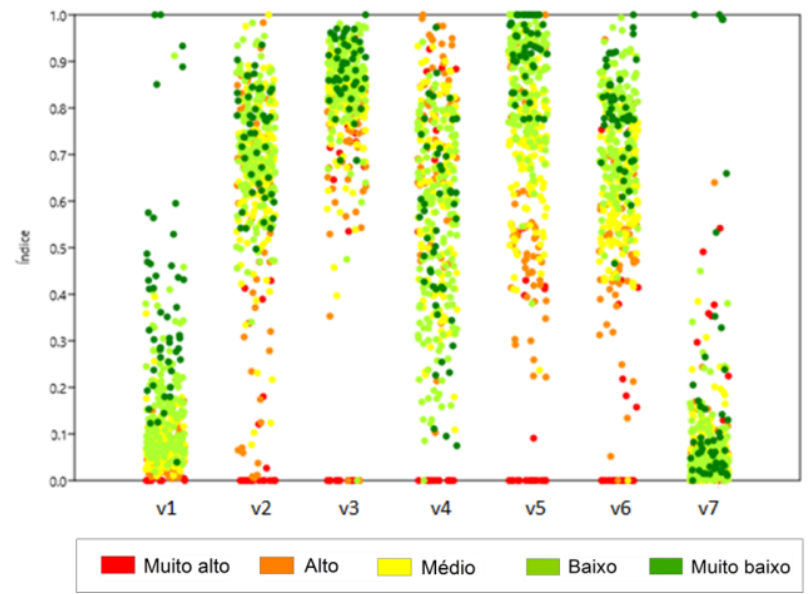

(b)

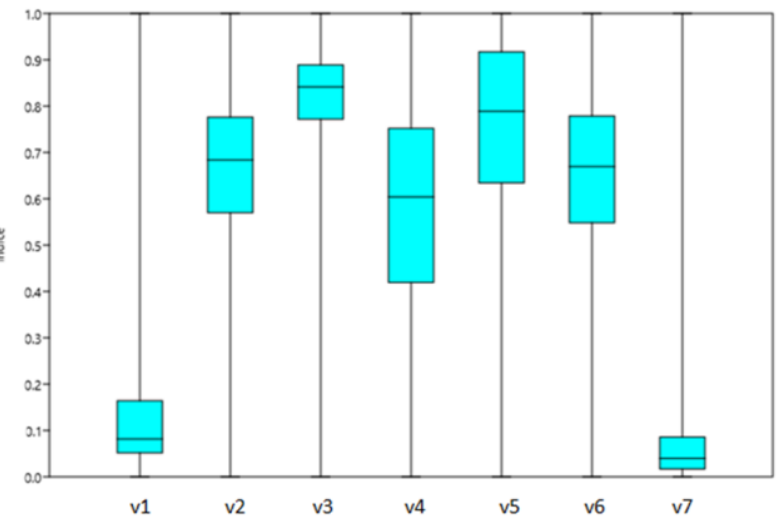


A analise multicriterial MANOVA apresentou o teste Wilks's Lambda $=0.07036, F=55.60$ e $p>0,05$, tais resultados confirmam, estatisticamente, que existe diferença intra e entre os grupos que foram definidos pelos agrupamentos categorizados em muito baixo, baixo, médio, alto e muito alto índice de segregação espacial estabelecidos neste estudo.

A análise de componentes principais (PCA) (Figura 6), mostra quais são os principais vetores determinantes dentro de cada um dos grupos de segregação espacial definidos. Observa-se que há um gradiente transicional entre os grupos, indo dos setores censitários mais segregados aos menos segregados, ainda, destaca-se que no grupo categorizado em "muito baixa" segregação espacial, as variáveis dominantes é o alto rendimento nominal do responsável pelo domicílio (v1) e o baixa proporcionalidade de responsáveis por domicílio entre 10 e 29 anos por setor. Tais variáveis tem comportamento oposto nos grupos de alta e muito alta segregação.

Figura 6 - Gráfica de dispersão dos dados pela Análise dos Componentes Principais (PCA) - (v1) Rendimento nominal do responsável pelo domicílio; (v2) Responsável por domicílio entre 10 e 29 anos por setor; (v3) Responsável por domicílio acima de 60 anos; (v4) Responsável por domicílio alfabetizados por setor; (v5) Crianças entre 0 e 5 anos por setor censitário; (v6) Domicílios quitados; (v7) Taxa de arborização estimada.

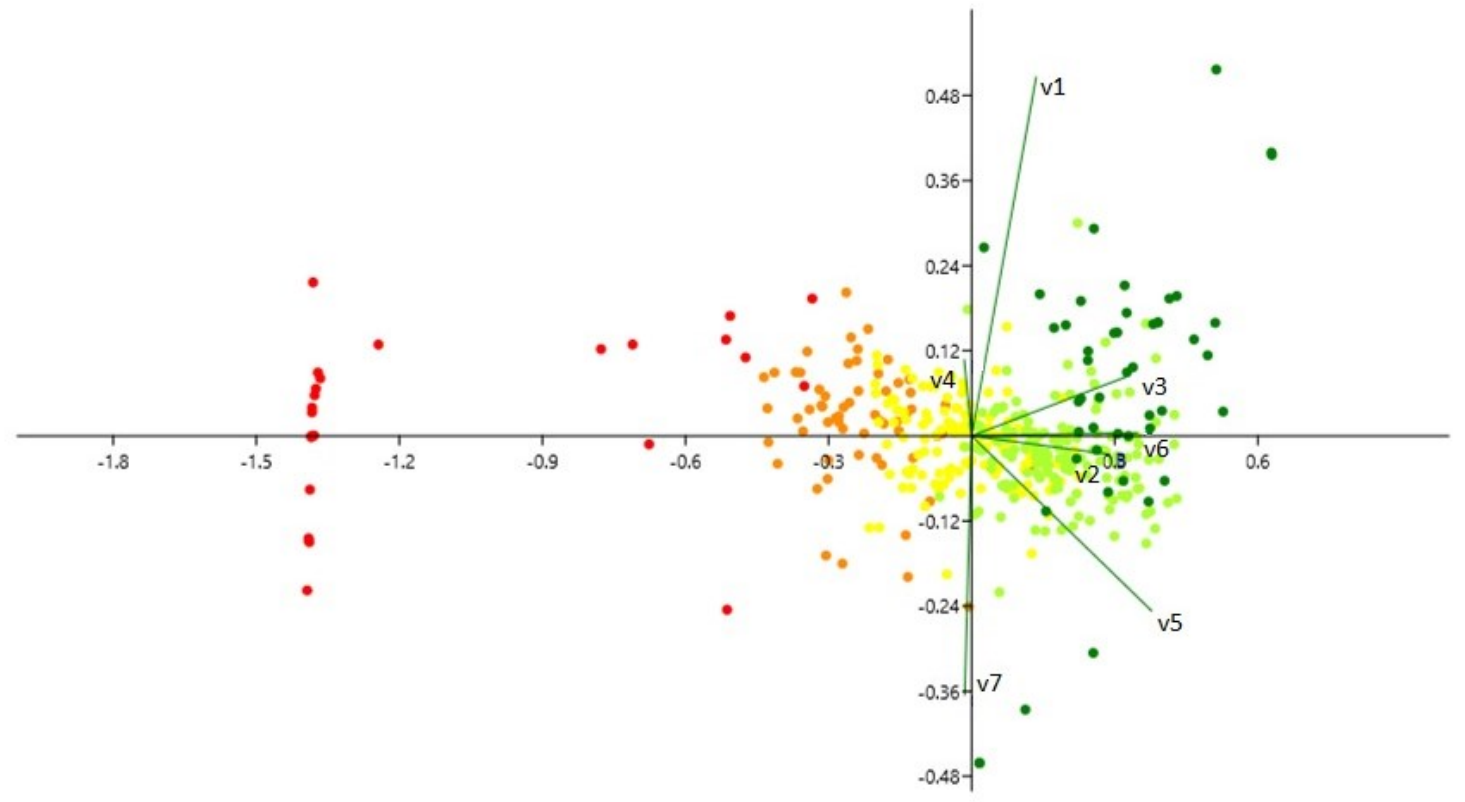

\section{CONSIDERAÇÕES FINAIS}

A análise da segregação espacial por meio da cartografia de síntese mostra que há um padrão de distribuição espacial do índice por setores censitários urbanos do município de Uberaba, MG, que pode ser interpretado por seus agrupamentos. Neste estudo, constatou-se a formação de espaços mais segregados e menos segregados na cidade, tendo a abordagem de seis variáveis demográficas e uma ambiental como fora descrito ao longo do texto.

Embora se trate de uma cidade de porte médio, esse resultado guarda semelhanças com o que ocorre em metrópoles e grandes cidades, corroborando a ideia de que para as cidades médias essa tendência se mantém. Embora duas variáveis não tenham apresentado um padrão centro-periferia, a saber: responsável por domicílio acima de 60 anos e a taxa de arborização estimada. Na integração sintética do índice, as demais variáveis foram preponderantes na determinação do resultado. A distribuição espacial do índice mostra um arranjo que, na comunicação cartográfica, denota um território desigual, com indicativo de segregação socioespacial.

A explicação para tal configuração espacial pode ser buscada nos fatores que constituíram os processos históricos da urbanização de Uberaba. A periferia da cidade, resulta de processos mais recentes de ocupação, com populações de menor renda. Nestes lugares, o quantitativo absoluto dos chefes de família mais jovens é alto. Assim, os chefes de família com 60 anos ou mais, em termos proporcionais, vão aparecer com taxas mais baixas nestes locais, muito provavelmente, devido a essa característica. Em contrapartida, nas áreas centrais, a população idosa absoluta é maior, o que tende a resultar, também, em

$\begin{array}{lllll}\text { Caminhos de Geografia } \quad \text { Uberlândia-MG } & \text { v. 22, n. } 80 \quad \text { abr./2021 } & \text { p. 297-308 } & \text { Página } 306\end{array}$


um quantitativo absoluto maior de domicílios com casais idosos, neste quesito, o índice tenderá ao padrão inverso do modelo visto em outra pesquisa (MARTINES et. al., 2019), com centro menos segregado em relação à periferia.

Com relação aos enclaves de alta e muito alta segregação espacial em regiões próximas ao centro, o processo histórico de formação aponta para décadas mais antigas. Para a compreensão destas áreas, faz-se necessário traçar o quadro das políticas relacionadas à ocupação desses territórios, contextualizando o acesso à terra e o perfil socioeconômico da população que hoje ali habita.

\section{REFERÊNCIAS}

COSTA, M. A.; MARGUTI, B. O. Atlas da vulnerabilidade social nos municípios brasileiros. Brasília: IPEA; 2015.

CUNICO, C. e OKA-FIORI, C.. O estado de normalidade e o estado de exceção diante da importância das categorias de "vulnerabilidade", "risco" e "resiliência". Caminhos de Geografia, Uberlândia, v15, n.52, p. 1-20, dez. $2014 . \quad$ Disponível em http://www.seer.ufu.br/index.php/caminhosdegeografia/article/download/24840/15956/. Acesso em 25 de fevereiro de 2020.

FRANÇA, V. O.; STRAUCH, J. C. M.; AJARA, C. 2014. Método Dasimétrico Inteligente: uma aplicação na mesorregião metropolitana de Belém. Revista Brasileira de Cartografia, v. 6, n. 66/6 disponível em http://www.lsie.unb.br/rbc/index.php/rbc/article/view/863. Acesso em 25 de fevereiro de 2020.

FUNDAÇÃO SEADE. Índice paulista de vulnerabilidade social - IPVS: espaços e dimensões da pobreza nos municípios do estado de São Paulo. 2010.

GOERL, R. F.; KOBIYAMA, M.; PELLERIN, J. R. G. M. Proposta metodológica para mapeamento de áreas de risco a inundação: estudo de caso do município de Rio Negrinho-SC. Boletim de Geografia, v. 30, n. 1, p. 81-100, 2012. https://doi.org/10.4025/bolgeogr.v30i1.13519

GOMES, M. T. S. Cidades médias, novos espaços produtivos e reestruturação do espaço urbano em Uberaba-MG. Confins. v.25, 2015. Disponível em: http://journals.openedition.org/confins/10407. Acesso em 22 fevereiro de 2020. https://doi.org/10.4000/confins.10407

INPE (Instituto Nacional de Pesquisas Espaciais). Manuais: Tutorial de geoprocessamento do SPRING. INPE, Sao José dos Campos, 2008.

JENKS, G. F. The data model concept in statistical mapping. International yearbook of cartography, v. 7, p. 186-190, 1967.

LEFEBVRE, H. O Direito à Cidade. São Paulo: Centauro. 2001. 143p

MARTINELLI, M. Cartografia ambiental: um mapa de síntese. Confins, 35, 2018. Disponível em http://journals.openedition.org/confins/13273. Acesso em 02 de março de 2020.

MARTINES, M. R.; TOPPA, R. H.; Cavagis, A.; FERREIRA, R. V. ; KAWAKUBO, F. S.; MORATO, R. G. Spatial Analysis to Identify Urban Areas with Higher Potential for Social Investment. Journal of Geographic Information System, v. 09, p. 591-603, 2017. https://doi.org/10.4000/confins.13273

MARTINES, M. R. MORATO, R. G; KAWAKUBO, F. S.; FERREIRA, R. V.; TOPPA, R. H. Análise dos aspectos demográficos e socioambientais para elaboração de um Índice de Segregação Espacial Urbana. Confins, n.40, 2019. Disponível em: http://journals.openedition.org/confins/19636. Acesso em 05 março de 2020.

QUEIROZ FILHO, A. P.; MARTINELLI, M. Cartografia de análise e de síntese na geografia. Boletim Paulista de Geografia, São Paulo, $\mathrm{n}^{\circ}$ 87, p. 7-44, 2007. Disponível em: https://www.agb.org.br/publicacoes/index.php/boletim-paulista/article/view/694/576. Acesso em 01 mar 2020.

ROUSE, J. W.; HAAS, R. H.; SCHELL, J. A.; DEERING, D. W. Monitoring vegetation systems in the Great Plains with ERTS. Third ERTS Symposium, NASA SP-351 I:309-317, 1974.

SANTOS, M. A urbanização brasileira. 5. ed. São Paulo: Edusp, 2005.

SCOTT, D. W. Sturges' rule. Wiley Interdisciplinary Reviews: Computational Statistics, v. 1, n. 3, p. 303306, 2009. https://doi.org/10.1002/wics.35

Caminhos de Geografia

$\begin{array}{lll}\text { v. } 22, \text { n. } 80 & \text { abr. } / 2021 & \text { p. } 297-308\end{array}$

Página 307 
TODOROV, V.; FILZMOSER, P. Robust Statistic for the One-way MANOVA. Computational Statistics \& Data Analysis, v. 54, n. 1, p. 37-48, 2010. https://doi.org/10.1016/j.csda.2009.08.015

VIEIRA, A. B; MELAZZO, E. S. Introdução ao conceito de segregação socioespacial. Formação (Presidente Prudente), 1, (10), 161-173. 2003. Disponível em http://revista.fct.unesp.br/index.php/formacao/article/view/1118/1123. Acesso em 20 de fevereiro de 2020.

VILLAÇA, F. J. M. Espaço intra-urbano no Brasil. São Paulo: Studio Nobel/Fapesp/ Lincoln Institute, 2001, 379p.

WONG, D. W. From aspatial to spatial, from global to local and individual: Are we on the right track to spatialize segregation measures? In: Recapturing space: New middle-range theory in spatial demography. Springer, Cham, 2016. p. 77-98. https://doi.org/10.1007/978-3-319-22810-5 5

WRIGHT, J. K. A method of mapping densities of population: With Cape Cod as an example. Geographical Review, v. 26, n. 1, p. 103-110, 1936. https://doi.org/10.2307/209467

YAO, J.; WONG, D. W.; BAILEY, N.; MINTON, J. Spatial segregation measures: A methodological review. Tijdschrift voor Economische en Sociale Geografie, 110(3), 235-250, 2018. https://doi.org/10.1111/tesg.12305

Recebido em: 24/05/2020

Aceito para publicação em: 18/03/2021 